\title{
Using differential phase for 3D localization of tracer particles in digital inline holographic microscopy PIV/PTV (DIHM-PIV/PTV)
}

\author{
A. Ahmed ${ }^{1 *}$, B. Sun ${ }^{1}$, V. J. Cadarso ${ }^{2}$, J. Soria ${ }^{1}$ \\ ${ }^{1}$ Laboratory for Turbulence Research in Aerospace \& Combustion (LTRAC) \\ 2 Applied Micro and Nanotechnology Lab (AMNTL) \\ Department of Mechanical and Aerospace Engineering, Monash University (Clayton Campus), VIC 3800, \\ Australia \\ *asif.ahmed@monash.edu
}

\begin{abstract}
Digital inline holographic microscopy PIV/PTV (DIHM-PIV/PTV) has the ability to provide 4-dimensional (4D), i.e. time-resolved, 3-component 3-dimensional (3C-3D) flow measurement with high spatial and temporal resolution, compact optical setup and minimal calibration Sun et al. (2020) compared to most other volumetric techniques such as tomo-PIV, defocusing PIV, etc. Despite all these advantages DIHMPIV/PTV has not yet developed into a standard laboratory tool due to some major limitations such as the extended depth-of-focus (DOF) problem and the virtual image effect which cause artefacts in the standard reconstruction volume limiting the seeding concentration and thus the achievable velocity spatial resolution. In order to mitigate the above-mentioned limitations we present a novel particle localization and extraction methodology which allows the minimization of these artefacts from the standard reconstruction and perform PIV/PTV analysis on the particle volume fields only. The proposed algorithm is based on the differential phase, which is the axial phase shift of the object wave compared to the reference plane wave propagation.

When micron-sized dielectric spheres are used as seeding particles (e.g. $1 \mu \mathrm{m}$ polystyrene microsphere in the present study) illuminated with a plane reference wave, a spatially strong confinement of the electromagnetic field, known as a photonic nanojet Heifetz et al. (2009), is observed on the shadow side of the particle as a result of Mie scattering of the incident wave from the isolated microspheres. This spatial light confinement does not occur exactly at the centre of the microparticles and is observed at a certain axial distance from the centre that depends on the numerical aperture of the imaging system. This phenomenon makes it very challenging to determine the axial location of the seeding particles from the intensity of the reconstruction volume. However, due to this spatial light confinement the object wave goes through a transition from a converging to a diverging wavefront and thus, a transition from positive to negative differential phase, which can be easily obtained from the phase of the complex reconstruction volume. This phase change has been exploited in the present study to localize the seeding particles within the reconstruction volume and extract the particle fields eliminating all the artefacts. Rigorous numerical simulations have been carried out using Mie scattering theory including the effect of limited numerical aperture of the imaging system to generate synthetic holograms Ahmed et al. (2020). The reconstruction volume obtained from the synthetic hologram shows excellent agreement with the one obtained from experiment, verifying the evolution of the photonic nanojet and transition in differential phase within the reconstruction volume.

A flowchart of the proposed methodology for hologram processing and extracting the 3D particle field from the reconstruction volume for PIV/PTV analysis is shown in Fig. 1. First, we calculate a background image from a sequence of recorded holograms. Then we use the background image to preprocess the raw recorded hologram that yields two new holograms, namely, the object hologram (using background subtraction) and the normalised hologram (using background division). Background subtraction removes the DC reference wave from the raw hologram and therefore, the reconstruction volume generated using the object hologram will only have the scattered object field. Thus, the object hologram is used to reconstruct the intensity volume which is then used to determine the $(x, y)$ positions and diameter of the particles within the volume. However, this intensity volume does not provide the accurate $z$ position of the seeding particles due to the above-mentioned fact that the peak intensity of the particle occurs at an axial distance away from the
\end{abstract}




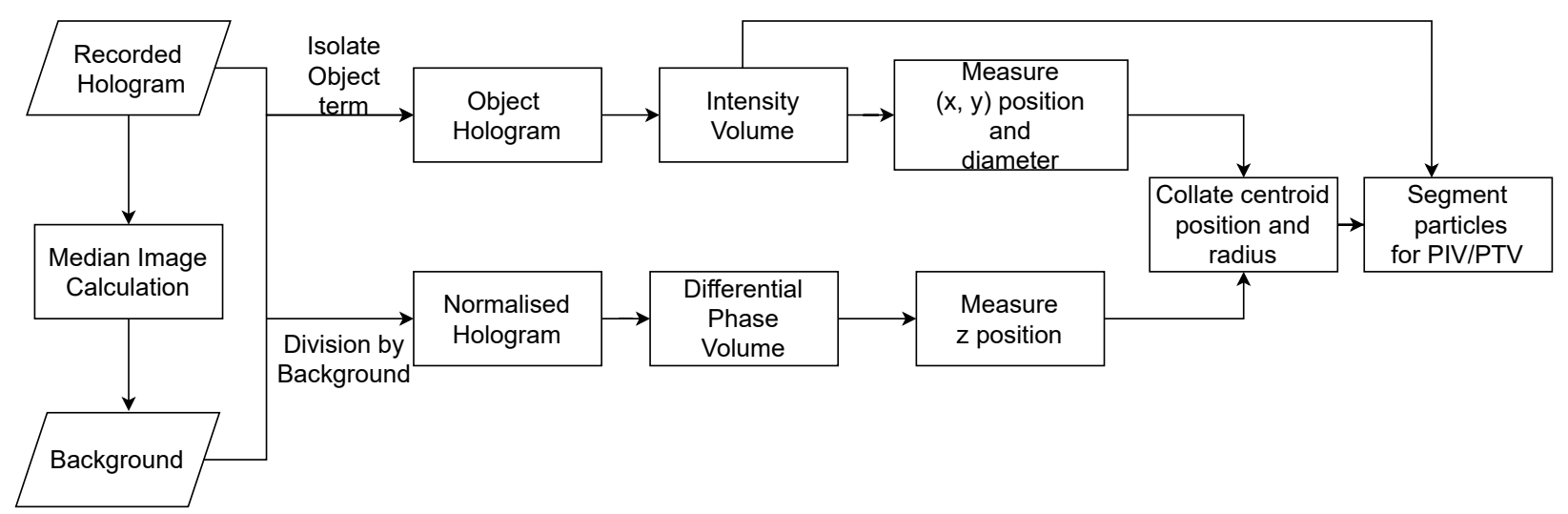

Figure 1: Flowchart of the proposed methodology for hologram processing and extracting the 3D particle field for PIV/PTV analysis

particle centre because of the evolution of the photonic nanojet. Hence, the normalised hologram is used to reconstruct the differential phase volume which is used to determine the $z$ position of the seeding particles. Background division improves the SNR of the hologram, however, it does not remove the DC reference wave. Therefore, the resulting reconstruction volume generated using the normalised hologram has both the reference wave, as well as the scattered object wave. As a result, the constructive and destructive interference of these two waves causes the change in differential phase which allows the determination of the accurate $z$ position of the seeding particles. In this new proposed method, the differential phase volume obtained from the experimental hologram is cross-correlated with the one is obtained from the simulated hologram of known particle position for the accurate localization of the seeding particles in the axial direction.

Finally, the $(x, y)$ positions and the diameter obtained from the intensity volume and the $z$ position obtained from the differential phase volume are used to yield the entire particle fields. Once a sequential pair of 3D volumes with particle fields is reconstructed these can be analysed using 3D cross-correlation analysis or a hybrid cross-correlation PIV/PTV to reveal the 3C-3D fluid velocity field Soria et al. (2014).

\section{Acknowledgements}

This research was supported by the Australian Government through the Australian Research Council's Discovery Projects funding scheme. Asif Ahmed gratefully acknowledge the support through an Australian Government Research Training Program (RTP) Scholarship.

\section{References}

Ahmed A, Sun B, Cadarso V, and Soria J (2020) 3d localization of the tracer particles in digital inline holographic microscopy piv/ptv: do the bright regions in the intensity reconstruction volume really correspond to the tracer particles?. Bulletin of the American Physical Society

Heifetz A, Kong SC, Sahakian AV, Taflove A, and Backman V (2009) Photonic nanojets. Journal of computational and theoretical nanoscience 6:1979-1992

Soria J, Atkinson C, and Buchmann N (2014) Hybrid PIV-Particle Tracking Technique Applied to High Reynolds Number Turbulent Boundary Layer Measurements. in 67th Annual Meeting of the APS Division of Fluid Dynamics, San Francisco, United States, Nov. 23-25.

Sun B, Ahmed A, Atkinson C, and Soria J (2020) A novel 4d digital holographic PIV/PTV (4d-DHPIV/PTV) methodology using iterative predictive inverse reconstruction. Measurement Science and Technology $31: 104002$ 